\title{
Organhaftung, EuGVO und Rom I-VO
}

\section{Zugleich Besprechung der Entscheidung EuGH NZG 2015, $1199 \mathrm{ff}^{*}$}

\author{
Dr. Leonhard HÜBner, MJur (Oxon), Heidelberg***
}

Inhaltsübersicht

ZGR 2016, 897-914

I. Die Entscheidung des EuGH _. . . . . . . . . . . . . . . . . . . . . . . . . 899

1. Sachverhalt . . . . . . . . . . . . . . . . . . . . . . . 899

2. Entscheidung des EuGH . . . . . . . . . . . . . . . . 900

II. Geschäftsleiter als Arbeitnehmer i.S.d. EuGVO . . . . . . . . . . . . . . . . . . 901

1. GmbH-Geschäftsführer als Arbeitnehmer i.S.d. EuGVO . . . . . . . . . . . 901

2. Auswirkungen von „Ferho" auf die Rom I-VO . . . . . . . . . . . . . . . . . 906

3. Konsequenzen aus „Ferho“ für andere Formen der Geschäftsleitung . . . . 909

III. Vertragsgerichtsstand . . . . . . . . . . . . . . . . . . . . 911

IV. Deliktsgerichtsstand . . . . . . . . . . . . . . . . . . . . 912

V. Zusammenfassung in Thesen . . . . . . . . . . . . . . . . . . . . . 913

Sind die Geschäftsleiter von Kapitalgesellschaften als Arbeitnehmer einzuordnen? Diese Frage wird im europäischen und deutschen Recht unterschiedlich beurteilt. Anders als das deutsche Recht, das zwischen Anstellungs- und Bestellungsverhältnis trennt, verfolgt der EuGH den Ansatz einer Gesamtbetrachtung der Verhältnisse von Geschäftsleiter und Gesellschaft. Die aktuelle Entscheidung des EuGH bestätigt dieses autonome Verständnis des europäischen Begriffs des Arbeitnehmers in Bezug auf Geschäftsleiter. Der Gerichtshof überträgt darin seine Rechtsprechung zu den arbeitsrechtsspezifischen Richtlinien auf das Europäische Zivilprozessrecht. Offen bleibt, ob, und falls ja, wie diese Rechtsprechung auf die Rom I-VO ̈̈bertragen werden kann.

European and German law are divided on the question whether managing directors of limited liability companies can be classified as employees. German law distinguishes between the service contract between the managing director and the company and the rights and obligations vis-à-vis the company that follow directly from company law. The ECJ, in contrast, follows an approach of an overall assessment of these relationships. The current judgment of the ECJ confirms this autonomous understanding of the European term of the "employee" as regards directors. The Court of Justice applies its case law relating to employment law specific Directives to the European law of civil procedure. It remains open, however, whether and (if yes) how this case law can be transferred to the Rome I-Regulation.

* EuGH (3. Kammer), Urt. v. 10.9.2015 - C-47/14 (Holterman Ferho Exploitatie ua/ Spies von Büllesheim).

** Der Autor ist Habilitand und Wissenschaftlicher Mitarbeiter am Institut für ausländisches und internationales Privat- und Wirtschaftsrecht an der Universität Heidelberg (Lehrstuhl Professor Dr. Marc-Philippe Weller). Er dankt Professor Dr. Christoph A. Kern, Ll.M. (Harvard), Professor Dr. Marc-Philippe Weller und Akad. Rat Dr. Adam SAgan, MJur (Oxon) für wertvolle Hinweise und Anmerkungen. 
VO (EG) Nr. 44/2001 Artikel 5 Nr. 1, 3, 18 I, 20 I, 60 EuGVO a.F.

1. Die Bestimmungen von Kapitel II Abschnitt 5 (Art. 18 bis 21) der Verordnung (EG) Nr. 44/2001 des Rates vom 22. Dezember 2000 über die gerichtliche Zuständigkeit und die Anerkennung und Vollstreckung von Entscheidungen in Zivil- und Handelssachen sind dahin auszulegen, dass sie in einer Situation wie der des Ausgangsverfahrens, in der eine Gesellschaft eine Person, die als ihr Direktor und Geschäftsführer tätig war, verklagt, um die von dieser Person in Wahrnehmung ihrer Aufgaben begangenen Fehler feststellen zu lassen und Schadensersatz zu erlangen, der Anwendung von Art. 5 Nrn. 1 und 3 der Verordnung entgegenstehen, sofern diese Person in ihrer Eigenschaft als Direktor und Geschäftsführer während einer bestimmten Zeit der Gesellschaft nach deren Weisung Leistungen erbrachte und dafür als Gegenleistung eine Vergütung erhielt; dies $\mathrm{zu}$ prüfen ist Sache des vorlegenden Gerichts.

2. Art. 5 Nr. 1 der Verordnung Nr. 44/2001 ist dahin auszulegen, dass eine Klage, die eine Gesellschaft gegen ihren ehemaligen Geschäftsführer erhebt, weil er die ihm obliegenden gesellschaftsrechtlichen Verpflichtungen verletzt haben soll, unter den Begriff „Vertrag oder Ansprüche aus einem Vertrag“ fällt. Mangels abweichender Angaben in der Satzung der Gesellschaft oder in einem anderen Dokument ist es Sache des vorlegenden Gerichts, den Ort zu bestimmen, an dem der Geschäftsführer seine Tätigkeiten zur Erfüllung des Vertrags tatsächlich überwiegend erbrachte, sofern die Erbringung der Dienstleistungen an diesem Ort nicht dem Willen der Parteien, wie er sich aus den zwischen ihnen geschlossenen Vereinbarungen ergibt, zuwiderlief.

3. Unter Umständen wie denen des Ausgangsverfahrens, die darin bestehen, dass eine Gesellschaft ihren ehemaligen Geschäftsführer wegen einer ihm zur Last gelegten unerlaubten Handlung verklagt, ist Art. 5 Nr. 3 der Verordnung Nr.44/2001 dahin auszulegen, dass eine solche Klage eine unerlaubte Handlung oder eine ihr gleichgestellte Handlung betrifft, wenn das dem Geschäftsführer zur Last gelegte Verhalten nicht als Verletzung seiner gesellschaftsrechtlichen Verpflichtungen angesehen werden kann, was zu prüfen Sache des vorlegenden Gerichts ist. Das vorlegende Gericht hat auf der Grundlage der tatsächlichen Umstände der Rechtssache den engsten Anknüpfungspunkt mit dem Ort des für den Schaden ursächlichen Geschehens und dem Ort der Verwirklichung des Schadenserfolgs zu ermitteln.

EuGH (3. Kammer), Urt. v. 10.9.2015 - C-47/14

(Holterman Ferho Exploitatie ua/Spies von Büllesheim) 


\section{Die Entscheidung des EuGH}

Die Tragweite der vorliegenden Entscheidung verdeutlicht eine Aussage von Stephan Harbarth und Carl Friedrich Nordmeier aus dem Jahr 2009:

„Es erscheint unbillig, einem Geschäftsführer denselben Schutz wie einem Arbeitnehmer zuzubilligen, der nach Art. 20 Abs. 1 EuGVO [a.F.] vom Arbeitgeber nur vor den Gerichten seines Wohnsitzstaats verklagt werden kann."

Mit dem vorliegenden Judikat hat der EuGH diese Tür jedoch weit geöffnet. Im Kern geht es bei diesem Urteil um die Frage, an welchem Gerichtsstand der EuGVO eine Gesellschaft ihren Geschäftsführer wegen der Verletzung seiner vertraglichen, organschaftlichen und deliktischen Pflichten verklagen kann, also um Fragen der internationalen Zuständigkeit. Den normativen Rahmen für die Entscheidung bilden vor allem Art. 22 Abs. 1 sowie Art. 7 Nr. 1 und 2 in der neuen Fassung der EuGVO.

\section{Sachverhalt}

Eine niederländische Kapitalgesellschaft in Rechtsform der BV verklagt ihren ehemaligen Geschäftsführer auf Schadenersatz vor niederländischen Gerichten. Der Wohnsitz des Geschäftsführers befindet sich in Deutschland. Die BV stützt sich auf drei Klagegründe: die Verletzung arbeitsvertraglicher, organschaftlicher und deliktischer Pflichten durch den Geschäftsführer.

Der Geschäftsführer rügt die internationale Zuständigkeit der niederländischen Gerichte, da für Klagen gegen ihn als Arbeitnehmer ausschließlich die Gerichte in seinem Wohnsitzstaat zuständig seien. Der Hoge Raad (das höchste Gericht der Niederlande) legte dem EuGH daraufhin die Frage vor, ob trotz der verschiedenen Klagegründe der Anspruch exklusiv am Arbeitnehmergerichtsstand nach Art. 22 EuGVO geltend gemacht werden kann. Falls dies zu bejahen wäre, könnte der Beklagte nur in Deutschland verklagt werden. Daneben wollte der Hoge Raad geklärt wissen, ob die Haftung des Geschäftsführers einen Vertrags- und auch einen Deliktsgerichtsstand nach Art. 7 EuGVO begründen könnte. 


\section{Entscheidung des EuGH}

Der EuGH geht in seiner Entscheidung von einem Stufenverhältnis zwischen den Gerichtsständen des Arbeitsverhältnisses, des Vertrags- und des Deliktsrechts aus. Die jeweils folgende Stufe ist nur anwendbar, wenn die Voraussetzungen der vorherigen Stufe nicht gegeben sind. Der EuGH stellt klar, dass zur Bestimmung des Begriffs des Arbeitnehmers seine Rechtsprechung zur Arbeitnehmerfreizügigkeit ${ }^{2}$ und zu bestimmten Sekundärrechtsakten ${ }^{3}$ heranzuziehen ist. Ein Geschäftsführer sei dann Arbeitnehmer, wenn er während einer bestimmten Zeit Leistungen für eine Gesellschaft nach deren Weisungen und gegen Gegenleistung erbringt, zu dieser Gesellschaft in dauerhafter Beziehung steht und in einer bestimmten Weise in den Betrieb eingegliedert ist. ${ }^{4}$ Das „wesentliche Merkmal eines Arbeitsverhältnisses“ sei die Weisungsabhängigkeit. ${ }^{5}$ Zur Ermittlung des Unterordnungsverhältnisses müssten aber alle Umstände des Einzelfalls herangezogen werden. ${ }^{6}$ Sei der Geschäftsleiter als Arbeitnehmer i.S.v. Art. 22 Abs. 1 EuGVO anzusehen, scheide eine Geltendmachung der Ansprüche an einem anderen Gerichtsstand aus, da es sich bei den Bestimmungen des Abschnitts 5 um ausschließliche Gerichtsstände handele. ${ }^{7}$

Eine Klage könne nur am Vertragsgerichtsstand nach $\ 7$ Nr. 1 EuGVO eingereicht werden, wenn der Hoge Raad zu dem Ergebnis kommt, dass der Geschäftsführer seine Aufgaben nicht als Arbeitnehmer der Gesellschaft erfüllt habe. ${ }^{8}$ Die Verletzung gesellschaftsrechtlicher Pflichten durch den Geschäftsführer als Organ könnte auch unter den Begriff „Vertrag oder Ansprüche aus einem Vertrag" gefasst werden, da in diesen Fällen eine ebenso enge Verbindung wie zwischen Vertragsparteien bestehe. ${ }^{9}$ Ein etwaiger deliktischer Gerichtsstand komme neben einem vertraglichen nur in Betracht, wenn die Klage nicht an das vertragliche Rechtsverhältnis zwischen der Gesellschaft und dem Geschäftsführer anknüpft. ${ }^{10}$ Soweit zusätzlich eine vertragliche Haftung

2 EuGH, Rs. C-47/14 (Ferho), NZG 2015, 1199, 1201, Rdn. 45; vgl. dazu von Hein, IWRZ 2016, 29 f; KindLER, IPRax 2016, $115 f$ f; LÜTTRINGHAUs, EuZW 2016, 904 ff.

3 EuGH, Rs. C-66-85 (Lawrie-Blum), NVwZ 1987, 41 f.

4 EuGH, Rs. C-232/09 (Danosa), Slg. 2010, I-11405.

5 EuGH, Rs. C-47/14 (Ferho), NZG 2015, 1199, 1201, Rdn. 41; LÜTtringhaus, EuZW 2016, 904, 905.

6 EuGH, Rs. C-47/14 (Ferho), NZG 2015, 1199, 1201, Rdn. 46.

7 EuGH, Rs. C-47/14 (Ferho), NZG 2015, 1199, 1201f, Rdn. 44, 51.

8 EuGH, Rs. C-47/14 (Ferho), NZG 2015, 1199, 1202, Rdn. 51.

9 EuGH, Rs. C-47/14 (Ferho), NZG 2015, 1199, 1202, Rdn. 54; vgl. auch EuGH, Rs. C34/82 (Peters), BeckEuRS 1983, 105380, Rdn. 13.

10 EuGH, Rs. C-47/14 (Ferho), NZG 2015, 1199, 1202, Rdn. 54; vgl. auch EuGH, Rs. C548/12 (Brogsitter), NJW 2014, 1648, 1649, Rdn. 24-27. 
greife, schließe diese eine Klage an einem denkbaren Deliktsgerichtsstand aus. ${ }^{11}$

\section{Geschäftsleiter als Arbeitnehmer i.S. d. EuGVO}

Mit der Entscheidung Ferho hat der EuGH in seiner Antwort auf die erste Vorlagefrage klargestellt, unter welchen Voraussetzungen Geschäftsleiter unter den Begriff des Arbeitnehmers i.S.v. EuGVO fallen. Der Beitrag will nach kurzer Darstellung der deutschen Rechtslage zeigen, dass der Gerichtshof seine bisherige Rechtsprechung zu dem Begriff des Arbeitnehmers auf die EuGVO überträgt. Im Anschluss widmet sich der Beitrag der Frage, ob dieser Begriff auch auf das materielle Kollisionsrecht in der Rom I-VO übertragen werden kann, und welche Konsequenzen daraus zu ziehen sind (unter B.II.). Ferner wird kurz untersucht, welche anderen Leitungsorgane in der gesetzlichen Konzeption dem Arbeitnehmerbegriff unterliegen (unter B. III.). Soweit dies nicht der Fall ist, kommt stattdessen der Vertrags- (unter C.) oder der Deliktsgerichtsstand (unter D.) des Art. 7 Nr. 1 oder 2 EuGVO in Frage.

\section{GmbH-Geschäftsfübrer als Arbeitnehmer i.S. d. EuGVO}

\section{a) Trennungsprinzip im deutschen Recht}

Nach der nationalen zivilprozessualen und materiell-rechtlichen Doktrin ist der Geschäftsführer kein Arbeitnehmer. Für das deutsche Prozessrecht legt $\ 5$ Abs. 1 Satz 3 ArbGG dies fest. Auf den Geschäftsführer einer $\mathrm{GmbH}$ findet somit nicht das ArbGG, sondern das GVG Anwendung. Auch der BGH sieht den Geschäftsführer in ständiger Rechtsprechung nicht als Arbeitnehmer im materiell-rechtlichen Sinn an. ${ }^{12}$ Bei dem Anstellungsverhältnis handelt es sich um einen Dienstvertrag mit Geschäftsbesorgungscharakter. ${ }^{13}$ Es fehlt an dem notwendigen sozialen Abhängigkeitsverhältnis, das Voraussetzung für die Annahme eines Arbeitsvertrags ist. ${ }^{14}$ Zudem handelt der Geschäftsführer gemäß

11 EuGH, Rs. C-47/14 (Ferho), NZG 2015, 1199, 1203, Rdn. 71.

12 Cruz Villalón, ECLI:EU:C:2015:309 = BeckRS 2015, 432609, Rdn. 36 - Schlussanträge Ferho.

13 BGH NJW 2010, 2343; Raiser/Veil, Recht der Kapitalgesellschaften, 6. Aufl., 2015, $\$ 42$ Rdn. 43; vgl. für den Vorstand einer AG Langenbucher, Aktien- und Kapitalmarktrecht, 3. Aufl., 2015, $\$ 4$ Rdn. 16.

14 BGHZ 49, 30, 31. 
$\$ 35 \mathrm{GmbHG}$ als Vertreter der Gesellschaft gegenüber den Angestellten und Arbeitern. ${ }^{15}$ Das Weisungsrecht der Gesellschafter besteht hingegen nur in dem organschaftlich fundierten Bestellungsverhältnis, das nach der sog. Trennungstheorie ${ }^{16}$ grundsätzlich streng von dem Anstellungsverhältnis zu separieren ist.

\section{b) Gesamtbetrachtung im europäischen Recht}

aa) Die Vorgeschichte: „Danosa“ und „Balkaya“

Demgegenüber kann nach der Rechtsprechung des EuGH der Geschäftsführer einer Kapitalgesellschaft unter den autonomen Begriff des Arbeitnehmers im unionsrechtlichen Sinn subsumiert werden. Die vorliegende Entscheidung fügt sich in die bisherige Rechtsprechungslinie des EuGH ein. ${ }^{17}$

Grundlegend war die 2009 ergangene Entscheidung Danosa. ${ }^{18}$ Diese betraf die Frage, ob die Geschäftsführerin einer lettischen Kapitalgesellschaft, die mit einer deutschen $\mathrm{GmbH}$ vergleichbar ist, ${ }^{19}$ den Begriff der Arbeitnehmerin erfüllt und damit in den Schutzbereich des Kündigungsschutzes nach der Mutterschutzrichtlinie fällt. Für den Begriff der Arbeitnehmerin hat der $\mathrm{EuGH}$ seine Rechtsprechung zur Arbeitnehmerfreizügigkeit auf diese arbeitsrechtsspezifische Richtlinie und damit auf das Sekundärrecht übertragen. ${ }^{20}$ Nach Rdn. 51 seines Judikats in Danosa fällt ein Geschäftsführer dem ersten Anschein nach unter den Begriff des Arbeitnehmers, wenn

(i) er gegen Entgelt Leistungen gegenüber der Gesellschaft erbringt,

(ii) die ihn bestellt hat und in die er eingegliedert ist,

(iii) er seine Tätigkeit nach der Weisung oder unter der Aufsicht eines anderen Organs der Gesellschaft ausübt und

(iv) er jederzeit ohne Einschränkung von seinem Amt abberufen werden kann. ${ }^{21}$

15 BGHZ 49, 30, 31 .

16 Koch, in: Hüffer, AktG, 11. Aufl., 2014, $\$ 84$ Rdn. 2.

17 Vgl. von Hein, IWRZ 2016, 29, 30; Kindler, IPRax 2016, 115, 116; LÜtTringhaus, EuZW 2016, 904, 905.

18 EuGH, Rs. C-232/09 (Danosa), Slg. 2010, I-11405 = NJW 2011, 2343; dazu kritisch aus nationaler gesellschaftsrechtlicher Sicht KORT, NZG 2013, $601 \mathrm{ff}$.

19 Krafka, Münchener Komm. z. HGB, 3. Aufl., 2010, \13 e Rdn. 5; Pentz, in: Ebenroth/ Boujong/Joost/Strohn, Handelsgesetzbuch, 3. Aufl., 2014, $\ 13$ e Rdn. 12.

20 EuGH, Rs. C-232/09 (Danosa), Slg. 2010, I-11405, Rdn. 39.

21 EuGH, Rs. C-232/09 (Danosa), Slg. 2010, I-11405, Rdn. 51; vgl. zu den Voraussetzungen im Einzelnen Preis/SAgan, ZGR 2013, 26, $40 \mathrm{ff}$. 
Die Formulierung „dem ersten Anschein nach“ bezieht sich darauf, dass der EuGH stets darauf verweist, dass das Unterordnungsverhältnis anhand „aller Gesichtspunkte und aller Umstände, die die Beziehungen zwischen den Beteiligten kennzeichnen, ${ }^{22} \mathrm{zu}$ bestimmen ist. ${ }^{23}$ Zentrale Kriterien dürften regelmäßig die Weisungsabhängigkeit (in Abgrenzung zu selbstständigen Tätigkeiten, die unter die Niederlassungs- und Dienstleistungsfreiheit fallen ${ }^{24}$ ) und die jederzeitige Abberufbarkeit des Geschäftsführers sein.

Die Rechtsprechung aus Danosa hat der EuGH in dem Urteil in der Rechtssache Balkaya ${ }^{25}$ (ebenfalls aus 2015) für den Arbeitnehmerbegriff der Massenentlassungsrichtlinie bestätigt. Er hat klargestellt, dass er - anders als das deutsche Recht - nicht zwischen Anstellungs- und Bestellungsverhältnis ${ }^{26}$ unterscheidet. Stattdessen nimmt der Gerichtshof eine Gesamtbetrachtung des Verhältnisses von Geschäftsführer und Gesellschaft vor. Zur Ermittlung des Unterordnungsverhältnisses zieht er Elemente sowohl des Bestellungsals auch des Anstellungsverhältnisses heran. ${ }^{27}$ Dies zeigt sich in Bezug auf die Weisung und die jederzeitige Abberufbarkeit in Danosa und Balkaya. In diesem Zusammenhang weist der EuGH stets darauf hin, dass der Geschäftsführer als Organ der Gesellschaft den Weisungen eines anderen Organs (nicht der Gesellschaft als Vertragspartner im Anstellungsverhältnis) unterliegt und er aus dem Amt jederzeit abberufen (nicht sein Vertrag gekündigt) werden kann. Er unterscheidet also nicht zwischen den einzelnen Verhältnissen. Im Gegenteil: In Danosa war der Abberufungsbeschluss (also die Abberufung aus dem Organverhältnis), und nicht die Kündigung des Anstellungsvertrags Gegenstand der zweiten Vorlagefrage, wie sich aus den Antworten des EuGH ergibt. ${ }^{28}$ Dieser Befund bestätigt: Die nationale Dogmatik ist für den EuGH irrelevant. ${ }^{29}$ Deutlich wird dies auch an der bereits erwähnten Rdn. 46 in Ferho, wonach das Unterordnungsverhältnis anhand ,aller Gesichtspunkte und aller Umstände, die die Beziehungen zwischen den Beteiligten kenn-

22 EuGH, Rs. C-47/14 (Ferho), NZG 2015, 1199, 1201, Rdn. 46; ebenso schon EuGH, Rs. C-232/09 (Danosa), Slg. 2010, I-11405, Rdn. 46; EuGH, Rs. C-229/14 (Balkaya), BeckRS 2015, 80897, Rdn. 37.

23 In diesem Sinne auch PreIs/SAGAN, ZGR 2013, 26, 40.

24 Forsthoff, in: Grabitz/Hilf/Nettesheim, Das Recht der Europäischen Union, 57. Ergänzungslieferung August 2015, Art. 45 AEUV Rdn. 69; vgl. EuGH, Rs. C-107/94 (Asscher), NJW 1996, 2921, 2922, Rdn. 26.

25 EuGH, Rs. C-229/14 (Balkaya), BeckRS 2015, 80897.

26 EuGH, Rs. C-229/14 (Balkaya), BeckRS 2015, 80897, Rdn. 38; bereits in EuGH, Rs. C232/09 (Danosa), Slg. 2010, I-11405, Rdn. 42, 47; vgl. auch Preis/SAGAN, ZGR 2013, 26, 37.

27 Vgl. auch PreIs/SAGAN, ZGR 2013, 26, $42 \mathrm{f}$.

28 EuGH, Rs. C-232/09 (Danosa), Slg. 2010, I-11405.

29 EuGH, Rs. C-232/09 (Danosa), Slg. 2010, I-11405, Rdn. 39f f; EuGH, Rs. C-229/14 (Balkaya), BeckRS 2015, 80897, Rdn. 33, 41. 
zeichnen, “30 zu bestimmen ist. Damit nivelliert der Gerichtshof die deutsche Trennungstheorie, die zwischen Anstellungs- und Bestellungsverhältnis trennt. Im Ergebnis ist also von einer Gesamtbetrachtung des EuGH auszugehen. ${ }^{31}$

\section{bb) Die Innovation durch „Ferbo“}

Mit dieser Entscheidung überträgt der EuGH seine Rechtsprechung zu dem unionalen Arbeitnehmerbegriff auf die EuGVO. Daraus folgt für Organhaftungsklagen (soweit das Organ unter den unionalen Arbeitsnehmerbegriff fällt) ein ausschließlicher Gerichtsstand und damit eine Zuständigkeitskonzentration $^{32}$ am Wohnsitz des beklagten Geschäftsführers nach Art. 22 EuGVO. $\mathrm{Da}$ es sich häufig um Geldleistungsansprüche gegen den Arbeitnehmer handeln wird, erscheint diese Regelung im Hinblick auf ein sich möglicherweise anschließendes Vollstreckungsverfahren auch sinnvoll. ${ }^{33}$ Während der EuGH dieses Ergebnis im Wesentlichen aus seiner bisherigen Rechtsprechung entwickelt, wird es auch durch systematische und teleologische Erwägungen gestützt.

\section{aaa) Systematische Auslegung}

Der EuGH schlägt in der Entscheidung Ferho die Brücke von spezifisch arbeitsrechtlichen Richtlinien zu allgemein zivilrechtlichen Regeln und überträgt diese Rechtsprechungslinie auf die EuGVO. Dies stellt der EuGH auch in Rdn. 42 seines Judikats explizit klar. ${ }^{34}$ Es handelt sich um einen Fall rechtsaktübergreifender Begriffsbildung ${ }^{35}$ im Hinblick auf den Arbeitnehmerbegriff. In systematischer Hinsicht fördert der EuGH damit die Einheit der Rechtsordnung und unterstützt den Kohärenzanspruchs des Unionsgesetzgebers. ${ }^{36}$ Der Gerichtshof konturiert zunehmend einen einheitlichen und autonomen unionalen Begriff des Arbeitnehmers. ${ }^{37}$

Im Sekundärrecht ist aber zwischen solchen Rechtsakten, die zur Bestimmung des Arbeitnehmerbegriffs auf das Recht des jeweiligen Mitgliedstaates ver-

30 EuGH, Rs. C-47/14 (Ferho), NZG 2015, 1199, 1201, Rdn. 46; ebenso schon EuGH, Rs. C-232/09 (Danosa), Slg. 2010, I-11405, Rdn. 46; EuGH, Rs. C-229/14 (Balkaya), BeckRS 2015, 80897, Rdn. 37.

31 Vgl. Mankowski, RIW 2015, 821, 823.

32 Mankowski, in: Rauscher, EuZPR/EuIPR, Band I, 4. Aufl., 2016, Art. 22 Brüssel IaVO, Rdn. 1.

33 Mankowski, aaO (Fn. 32), Art. 22 Brüssel Ia-VO, Rdn. 3.

34 EuGH, Rs. C-47/14 (Ferho), NZG 2015, 1199, 1201, Rdn. 42.

35 Vgl. LÜtTringhaus, RabelsZ 77 (2013), $31 \mathrm{ff}$.

36 Riesenhuber, in: ders., Europäische Methodenlehre, 3. Aufl., 2015, $\$ 10$ Rdn. 24.

37 Dagegen Jaeger, Münchener Komm. z. GmbHG, 2. Aufl., 2016, \35 Rdn. 280. 
weisen, und solchen Rechtsakten zu trennen, die keinen Verweis enthalten. ${ }^{38}$ In dem Urteil in der Rs. Union syndicale Solidaires Isère lässt der EuGH einen fehlenden Hinweis auf nationales Recht dafür ausreichen, dass der Arbeitnehmerbegriff in der konkreten Richtlinie unionsrechtlich zu verstehen ist. ${ }^{39}$ Die Differenzierung verläuft daher nicht zwischen den verschiedenen Arbeitnehmerbegriffen an der Trennlinie zwischen europäischem Primär- und Sekundärrecht, sondern zwischen einem unionalen und einem mitgliedstaatlichen Arbeitnehmerbegriff. ${ }^{40}$

\section{bbb) Telelogische Auslegung}

Das Ergebnis dieser systematischen Erwägungen wird auch durch die teleologische Auslegung des Art. 22 EuGVO gestützt. Der EuGH weist auch auf den Zweck dieser Vorschriften, nämlich den Schutz des Arbeitnehmers als „schwächere Partei“ des Arbeitsvertrags, hin. Diese Schutzrichtung ergibt sich aus den Erwägungsgründen 18 und 19 der EuGVO.

Auf den Schutz des Arbeitnehmers hatte der EuGH bereits in den Vorgängerurteilen Danosa und Balkaya abgestellt. In beiden Fällen hatte er auf das Gebot einer nicht zu engen Auslegung des Begriffs des Arbeitnehmers hingewiesen: in Danosa, um den Schutz der schwangeren und stillenden Beschäftigten nicht zu unterlaufen ${ }^{41}$ und in Balkaya, um den Schutz bei Massenentlassungen $\mathrm{zu}$ verstärken. ${ }^{42}$ Der Schutz des Arbeitnehmers ist also allen angesprochenen Sekundärrechtsakten als Fixpunkt der teleologischen Auslegung gemein.

\section{ccc) IPR: Weisung und Abberufbarkeit als Vorfrage des Gesellschaftsstatuts}

Wie bereits ausgeführt, können das für den EuGH relevante Weisungsrecht und die jederzeitige Abberufbarkeit aus dem gesellschaftsrechtlichen Organverhältnis folgen. Sie müssen nicht zwingend aus dem Dienstvertrag resultieren. Das Bestehen der beiden Voraussetzungen kann in diesem Fall nur unter Rückgriff auf das Gesellschaftsstatut der jeweiligen Gesellschaft zu ermitteln sein. An dieser kollisionsrechtlichen Notwendigkeit vermag auch die vom EuGH vorgenommene Gesamtbetrachtung, die sich auf die autonome Aus-

38 Vgl. SAgAn, in: Preis/Sagan, Europ. Arbeitsrecht, $\mathbb{1} 1$ Rdn. 107; Forst, EuZW 2015, 664, 665.

39 EuGH, Rs. C-428/09 (Union syndicale Solidaires Isère), BeckEuRS 2009, 507670, Rdn. $27 f$.

40 SAGAN, aaO (Fn. 38), $\ 1$ Rdn. 109; vgl. aber den eigenständigen Arbeitnehmerbegriff in Art. 3 lit. a Arbeitsschutzrahmenrichtlinie 89/391/EWG.

41 EuGH, Rs. C-232/09 (Danosa), Slg. 2010, I-11405, Rdn. 54.

42 EuGH, Rs. C-229/14 (Balkaya), BeckRS 2015, 80897, Rdn. 44. 
legung des Unionsrechts stützt, nichts zu ändern. Bei beiden Fragen handelt es sich um kollisionsrechtliche Vorfragen i.w.S. bzw. Erstfragen. ${ }^{43}$ Sie können sich auch in international-zivilverfahrensrechtlichen Vorschriften stellen. ${ }^{44}$ Maßgeblich ist das Gesellschaftsstatut, d.h. das auf die Gesellschaft anwendbare Recht. ${ }^{45}$

\section{Auswirkungen von „Ferbo" auf die Rom I-VO}

\section{a) Übertragbarkeit der Rechtsprechung des EuGH}

Mit der Schaffung eines unionalen Arbeitnehmerbegriffs stellt sich die Frage nach den Auswirkungen auf andere Sekundärrechtsakte. Besonders relevant erscheint dies für das materielle Kollisionsrecht in Form der Rom I-VO, d.h. den für Arbeitsverträge geltenden Art. 8. Den normativen Ansatzpunkt für die Übertragung der Rechtsprechung auf die Rom I-VO bildet Erwägungsgrund 7. Dieser normiert das Gebot der einheitlichen Auslegung von EuGVO und Rom I-VO. $\mathrm{Zu}$ überlegen ist daher, ob der Arbeitnehmerbegriff aus der EuGVO auf die Rom I-VO zu übertragen ist. Auf den ersten Blick sprechen teleologische Argumente für eine Übertragung. Denn Art. 8 Rom I-VO dient - wie auch die angesprochenen arbeitsrechtlichen Richtlinien - dem Schutz des Arbeitnehmers als schwächerer Vertragspartei, wie sich aus ihrem Erwägungsgrund $23^{46}$ ergibt. $^{47}$

\section{b) Konsequenzen der Gesamtbetrachtung des EuGH}

Verfolgt man den Ansatz der Gesamtbetrachtung aus Danosa, Balkaya und Ferho stringent weiter, könnte auch die Beurteilung des Organverhältnisses unter den Begriff des Arbeitsvertrags i.S.v. Art. 8 Rom I-VO fallen. Dies könnte wiederum zu erheblichen Abgrenzungsschwierigkeiten zwischen Gesellschafts- und Arbeitsvertragsstatut führen, soweit die jeweilige lex fori-wie das deutsche Recht - dem Trennungsprinzip folgt. ${ }^{48}$

43 Kindler, IPRax 2016, 115, 116f; Mankowski, RIW 2015, 821, 822; vgl. v. Hein, Münchener Komm. z. BGB, 6. Aufl., 2015, Einl. IPR, Rdn. 161.

45 KindleR, IPRax 2016, 115, 117.

46 Vgl. EuGH, Rs. C-29/10 (Koelzsch), EuZW 2012, 302, 305, Rdn. 46.

47 Ebenfalls für einen Gleichlauf von EuGVO und Rom I-VO in Bezug auf den Arbeitnehmerbegriff KindLER, IPRax 2016, 115; LÜTTRINGHAUs, EuZW 2015, 904, 906.

48 Vgl. zur niederländischen BV Rademakers/de Vries, in: Süß/Wachter, Handbuch des internationalen GmbH-Rechts, 2. Aufl., 2011, G. Rdn. 239. 
Diese Problematik soll an dem folgenden Beispiel veranschaulicht werden: Ein Geschäftsführer einer niederländischen BV mit Wohnsitz in Deutschland, der mit der BV einen deutschem Recht unterliegenden Anstellungsvertrag geschlossen hat, klagt sowohl gegen seine Abberufung aus dem Organverhältnis als auch gegen die Kündigung des Anstellungsvertrags.

Unter diesen Umständen stellt sich die Frage, welches Recht für die Beendigung der beiden Rechtsverhältnisse bei konsequenter Fortführung der Gesamtbetrachtung gilt. Auf die Kündigung des Anstellungsvertrags ist wegen der Rechtswahl deutsches Recht anwendbar. Unter Zugrundelegung der Gesamtbetrachtung des EuGH könnte man erwägen, die Abberufung oder sogar weitergehend das gesamte Organverhältnis dem Arbeitsvertragsstatut zuzuordnen. Das läge der Sache nach auf der Linie des Urteils in der Rechtssache Danosa, worin der EuGH den Schutz der arbeitsrechtlichen Mutterschutzrichtlinie auf das Bestellungsverhältnis ausdehnt. ${ }^{49}$ Grundsätzlich sind drei Lösungsansätze denkbar:

aa) Geltung von Art. 8 Rom I-VO auch im Bestellungsverhältnis

Das Bestellungsverhältnis könnte zunächst - wie das Anstellungsverhältnis ausschließlich dem Arbeitsvertragsstatut zugeordnet werden. In dem angeführten Beispiel wäre auf das Bestellungsverhältnis einer niederländischen BV zu ihrem in Deutschland wohnenden Geschäftsführer allein deutsches Arbeitsrecht anwendbar. Das Bestellungsverhältnis wäre dann nicht mehr Teil des Gesellschaftsstatuts. Diese Lösung wäre allerdings aus verschiedenen Gründen, die im Weiteren ausgeführt werden, schwer vorstellbar. Insbesondere geriete eine solche Auslegung des Arbeitnehmerbegriffs in Konflikt mit dem bisherigen Verständnis der Rom I-VO.

\section{bb) Strikte Trennung von Anstellungs- und Bestellungsverbältnis}

Daher könnte als zweiter Lösungsansatz nahe liegen, für die Zwecke der Rom I-VO entsprechend der deutschen Trennungstheorie zwischen dem Bestellungs- und Anstellungsverhältnis des Geschäftsführers zu unterscheiden. Die Bereichsausnahme des Art. 1 Abs. 2 lit. f) Rom I-VO ordnet die „innere Verfassung" der Gesellschaft dem Gesellschaftsstatut zu und nimmt sie aus dem Anwendungsbereich der Rom I-VO aus. Zu der „inneren Verfassung “ zählen nach bisherigem Verständnis sämtliche Binnenverhältnisse der Gesellschaft und damit auch die organschaftliche Beziehung zwischen der Gesellschaft und ihrem Geschäftsführer. ${ }^{50}$

50 Vgl. U. Magnus, in: Staudinger, IntVertrR, Neubearb. 2011, Art 1 Rom I-VO, Rdn. 85; Martiny, Münchener Komm. z. BGB, 6. Aufl., 2015, Art. 1 Rom I-VO, Rdn. 67. 
Voraussetzung für diese strikte kollisionsrechtliche Trennung von Anstellungs- und Bestellungsverhältnis wäre zwar die Überwindung des Gebots der einheitlichen Auslegung von EuGVO und Rom I-VO nach Erwägungsgrund 7. Dieser hat aber „nur“ die Funktion eines policy statement, das die Leitlinie für die telelogische Auslegung bietet. ${ }^{51}$ Gegenüber dem Erwägungsgrund setzt sich aber der verbindliche Art. 1 Abs. 2 lit. f) Rom I-VO durch. Das Organverhältnis (und damit auch Fragen betreffend die Rechtmäßigkeit der Abberufung) könnte deshalb in Überwindung des Gebots der einheitlichen Auslegung dem Gesellschaftsstatut unterfallen.

Ein anderes Verständnis ließe sich auch aufgrund des Begriffs der „Inneren Verfassung" der Gesellschaft nur schwer begründen. Unter den Begriff der „Verfassung“ fällt bei Kapitalgesellschaften, die ihrem Wesen nach fremdorganschaftlich organisiert sind, auch das Verhältnis Gesellschaft-Geschäftsleiter. Die Fremdorganschaft ist ein zentrales Charakteristikum der Kapitalgesellschaft. ${ }^{52}$ Zählt man das Bestellungsverhältnis im Rahmen der Rom I-VO nicht zu dem Gesellschaftsstatut, nimmt man einen wesentlichen Bestandteil der „inneren Verfassung“ aus dem Gesellschaftsstatut aus.

Problematisch wäre aber an diesem Ansatz, dass er die Gesamtbetrachtung des EuGH ignoriert. Es zeigt sich in der Danosa-Rechtsprechung, dass der Gerichtshof den arbeitsrechtlichen Kündigungsschutz der Mutterschutzrichtlinie auch im Bestellungsverhältnis anwenden möchte. ${ }^{53}$ Nimmt man diese Erkenntnis ernst, muss jedenfalls der arbeitsrechtliche Schutz, der sich aus dem Unionsrecht ergibt, auch in dem Bestellungsverhältnis sichergestellt werden. Dies kann eine strikte Trennung der Verhältnisse nicht leisten.

\section{cc) Doppelqualifikation}

Soweit man anerkennt, dass der EuGH in seiner Rechtsprechung keine Exklusivitätsthese zwischen Gesellschafts- und Arbeitsstatut aufstellt, wäre eine Doppelqualifikation ${ }^{54}$ des Bestellungsverhältnisses als gesellschafts- und arbeitsrechtlich zu erwägen. Das Gesellschaftsstatut könnte unter Hinweis auf Art. 8 Rom I-VO allein um den unionsrechtlich gebotenen Schutz des Arbeitnehmers ergänzt werden. $\mathrm{Zu}$ diesem Schutz würden allerdings nur die nationalen Vorschriften zählen, die auf europäischen Richtlinien wie der Arbeitszeit-, der Mutterschutz- oder der Massenentlassungsrichtlinie beru-

51 Köndgen, in: Riesenhuber, Europäische Methodenlehre, 3. Aufl., 2015, $\mathbb{1 0}$ Rdn. 51.

52 Drygala/Staake/Szalai, Kapitalgesellschaftsrecht, 2011, \1 Rdn. 7; Roth/Weller, Handels- und Gesellschaftsrecht, 7. Aufl., 2010, Rdn. 488.

53 EuGH, Rs. C-232/09 (Danosa), Slg. 2010, I-11405, Rdn. 74.

54 Vgl. v. Bar/Mankowski, Internationales Privatrecht, Band I, Allgemeine Lehren, 2. Aufl., 2003, $\$ 7$ Rdn. 178; Weller, Europäische Rechtsformwahlfreiheit und Gesellschafterhaftung, 2004, $274 \mathrm{ff}$. 
hen. ${ }^{55} \mathrm{Im}$ Ergebnis würde dies dazu führen, dass der Schutz dieser Richtlinien auch im Bestellungsverhältnis gelten müsste. ${ }^{56} \mathrm{Ob}$ daneben das sonstige genuin nationale Arbeitsrecht auf den Geschäftsführer anwendbar ist, wäre dann eine Frage des jeweiligen nationalen Rechts.

Aus Sicht des IPR darf eine solche Doppelqualifikation nur die ultima ratio darstellen. ${ }^{57}$ Der Charme der Doppelqualifikation besteht jedoch darin, dass sie - im Unterschied zu den anderen Optionen - alle angesprochenen Interessen und Normen in einen angemessenen Ausgleich bringt. Zum einen unterfiele das Bestellungsverhältnis wie bisher dem Gesellschaftsstatut. Zum anderen wäre gleichzeitig die Gesamtbetrachtung des EuGH berücksichtigt, indem Art. 8 Rom I-VO den Schutz des Arbeitnehmers im Bestellungsverhältnis koordiniert. Denkbar wäre es, den Arbeitnehmerschutz über Art. 8 Abs. 4 Rom I-VO akzessorisch an das Recht des Gesellschaftsstatuts anzuknüpfen. Dies würde zumindest auch zur Anwendung einer einzigen Rechtsordnung auf das Bestellungsverhältnis führen. Da es sich um europaweit harmonisiertes Recht zum Schutz des Arbeitnehmers handelt, ergibt sich so auch kein anderes Ergebnis als bei der Anwendung ausländischen Arbeitsrechts. Somit erscheint eine sinnvolle Koordinierung des arbeitsrechtlichen Schutzes im Bestellungsverhältnis über Art. 8 Rom I-VO möglich.

\section{dd) Zusammenfassung zur Rom I-VO}

Zusammenfassend ist festzuhalten, dass gegenwärtig ungeklärt ist, ob und wie der EuGH seine Rechtsprechung zu dem Arbeitnehmerbegriff auf die Rom IVO übertragen wird. Es wäre aber nicht überraschend, sollte der EuGH diesen auf Art. 8 Rom I-VO erstrecken. Bejaht man die Arbeitnehmereigenschaft, erscheint bei konsequenter Fortführung der Gesamtbetrachtung eine Doppelqualifikation des Bestellungsverhältnisses als gesellschafts- und arbeitsrechtlich am ehesten geeignet, um alle Interessen sachgerecht zu koordinieren.

\section{Konsequenzen aus „Ferho “ für andere Formen der Geschäftsleitung}

Im Folgenden sollen die Konsequenzen aus dem Urteil in der Rechtssache Ferho für andere Formen der Geschäftsführung gezogen werden. Der Beitrag behandelt insofern die gesetzlich vorgesehene Konstellation der Geschäftsführung.

55 SAGAN, aaO (Fn. 38), $\$ 1$ Rdn. 112.

56 Preis/SAGAN, ZGR 2013, 26, 36 („Mutterschutz im Bestellungsverhältnis?“).

57 v. Bar/Mankowski, aaO (Fn. 54), $\mathbb{7}$ Rdn. 178; v. Hein, aaO (Fn. 43), Einl. IPR, Rdn. 145. 


\section{a) Geschäftsfübrender Gesellschafter}

Nach Ferbo kann sich die Rechtslage ändern, wenn der Geschäftsführer auch Gesellschafter ist. ${ }^{58}$ Wenn der Geschäftsführer kraft Stimmmacht Einfluss auf die Abstimmung in der Gesellschafterversammlung nehmen kann, könnte an der notwendigen Weisungsabhängigkeit des Geschäftsführers fehlen. ${ }^{59} \mathrm{Er}$ müsste also Allein- Ein-Mann-GmbH), Mehrheits-, oder Minderheitsgesellschafter mit Sperrminorität sein. Daneben sind aber auch besondere gesellschaftsrechtliche Vereinbarungen neben der Satzung wie Stimmvereinbarungen oder andere Nebenabreden denkbar. Hier zeigt sich wieder, dass das Vorliegen eines Unterordnungsverhältnisses als Voraussetzung für den unionalen Arbeitnehmerbegriff stets unter Berücksichtigung aller Umstände des Einzelfalls zu ermitteln ist. ${ }^{60}$

\section{b) Vorstand einer AG/Leitungsorgan einer monistisch verfassten $S E$}

Demgegenüber ist der Vorstand einer AG nicht weisungsabhängig, wie sich aus $\ 76$ Abs. 1 AktG ergibt. Im Übrigen ist er auch nicht jederzeit, sondern nur aus wichtigem Grund abberufbar, vgl. $\ 84$ Abs. 3 AktG. ${ }^{61}$ Mangels Weisungsabhängigkeit kann er daher kein Arbeitnehmer im unionsrechtlichen Sinn sein. ${ }^{62}$

Das Leitungsorgan einer monistisch verfassten SE könnte hingegen unter den Arbeitnehmerbegriff fallen. ${ }^{63}$ Seine Mitglieder sind nach $\mathbb{} 40$ Abs. 5 SEAG jederzeit abberufbar, sofern die Satzung nichts anderes regelt. Daneben unterliegen sie gemäß $\ 44$ Abs. 2 SEAG einer Weisungsbindung im Innenverhältnis.

\section{c) Folgerungen}

Sofern die Mitglieder des Leitungsorgans Arbeitnehmer im unionsrechtlichen Sinn darstellen, fallen Klagen gegen sie unter den ausschließlichen internationalen Gerichtsstand vor den Gerichten ihres Wohnsitz-Mitgliedstaats i.S.v.

58 EuGH, Rs. C-47/14 (Ferho), NZG 2015, 1199, 1201, Rdn. 47.

59 JUNKER, NZA 2011, 950, 951.

60 LÜTTRINGHAUs, EuZW 2016, 904, 906.

61 KORT, NZG 2013, 601, 606.

62 LÜtTringhaus, EuZW 2016, 904, 906; von Hein, IWRZ 2016, 29, 30; anders aber ggf. im bei einer abhängigen AG im Vertragskonzern ( $\$ 308$ AktG) KIndLer, IPRax 2016, $115,116$.

63 Forst, EuZW 2015, 664, 667. 
Art. 22 Abs. 2, 23 EuGVO. Aufgrund der gebotenen Einzelfallbetrachtung kommt es auf die konkreten Verhältnisse in der jeweiligen Gesellschaft an. Erfüllen sie die Kriterien nicht, kommen die Gerichtsstände am Erfüllungsort aus Dienstvertrag nach Art. 7 Nr. 1 b) 2. Spiegelstrich EuGVO oder des Deliktsgerichtsstands nach Art. Art. 7 Nr. 2 EuGVO in Betracht. Mithin haben die anderen beiden Vorlagefragen Bedeutung für solche Geschäftsleiter, die nicht unter den Arbeitnehmerbegriff der EuGVO fallen. ${ }^{64} \mathrm{Zu}$ den fraglichen Geschäftsleitern zählen die Gesellschafter-Geschäftsführer mit bestimmendem Einfluss auf die Gesellschafterversammlung oder die Vorstandsmitglieder einer AG.

\section{Vertragsgerichtsstand}

In Beantwortung der zweiten Vorlagefrage stellt der EuGH fest, dass die Verletzung „gesellschaftsrechtlicher Verpflichtungen "65 eines Geschäftsführers unter den Begriff „Vertrag oder Ansprüche aus einem Vertrag“ i.S.v. Art. 7 Nr. 1 EuGVO zu fassen ist. Für diesen Schluss bemüht der EuGH zwei Argumente. Zum einen handele es sich bei der Verbindung zwischen Gesellschaft und Geschäftsführer um gegenseitige freiwillig eingegangene Verpflichtungen. Eine solche Bindung sei für ein Vertragsverhältnis charakteristisch, so dass das fragliche Verhältnis vertraglicher Natur sei. ${ }^{66}$ Zum anderen bestehe eine ebenso enge Verbindung zwischen Geschäftsführer und Gesellschaft wie zwischen zwei Vertragsparteien. ${ }^{67}$ Hinsichtlich des Erfüllungsortes sei auf Art. 7 Nr. 1 b) 2. Spiegelstrich EuGVO abzustellen, da es sich bei der charakteristischen Verpflichtung eines Geschäftsführers gegenüber der Gesellschaft um die „Erbringung einer Dienstleistung“ handele. Für die Ermittlung des Erfüllungsortes ${ }^{68}$ sei zunächst auf eine Klausel im Anstellungsvertrag abzustellen, sodann auf die Satzung oder andere Dokumente, die Regelungen zu Pflichten des Geschäftsleiters enthalten; seien daraus keine Hinweise ersichtlich, müsse das nationale Gericht den Ort ermitteln, an dem Geschäftsführer seine Tätigkeiten tatsächlich weit überwiegend erbrachte. ${ }^{69}$

64 Vgl. EuGH, Rs. C-47/14 (Ferho), NZG 2015, 1199, 1202, Rdn. 51.

65 EuGH, Rs. C-47/14 (Ferho), NZG 2015, 1199, 1201f, Rdn. 50. Damit sind die organschaftlichen Pflichten des Geschäftsführers gemeint.

66 EuGH, Rs. C-47/14 (Ferho), NZG 2015, 1199, 1202, Rdn. 52 f.

67 EuGH, Rs. C-47/14 (Ferho), NZG 2015, 1199, 1202, Rdn. 54; EuGH, Rs. C-34/82 (Peters), BeckEuRS 1983, 105380, Rdn. 13; vgl. dazu Weller, ZGR 2012, 606, 615.

68 Vgl. umfassend Wais, Der europäische Erfüllungsgerichtsstand für Dienstleistungsverträge.

69 EuGH, Rs. C-47/14 (Ferho), NZG 2015, 1199, 1202, Rdn. 52 f. 
Mit sehr knapper Argumentation begründet der EuGH, dass Ansprüche der Gesellschaft gegen den Geschäftsführer wegen der Verletzung gesellschaftsrechtlicher Pflichten unter Art. 7 Nr. 1 EuGVO fallen. Dabei stützt er sich auf sein Urteil in der Rechtssache Peters, worin er den Gerichtsstand des Erfüllungsortes nach Art. 7 Nr. 1 EuGVO für eine Klage eines niederländischen Vereins gegen ein deutsches Vereinsmitglied auf Zahlung aus einer für die Mitglieder bindenden Regelung bejahte. ${ }^{70}$ Es handelte sich aber um eine Klage zwischen Gesellschaft und Mitglied. Dieses Verhältnis lässt sich als Vertrag i.S.v. Art. 7 Nr. 1 EuGVO begreifen, da der rechtsgeschäftliche Vereinsbeitritt entsprechende Bindungen wie ein Austauschvertrag begründet. ${ }^{71}$ Die Rechtssache Ferbo betrifft hingegen die Klage der Gesellschaft gegen ihren ehemaligen Geschäftsführer. Dies betrifft ein anderes Binnenverhältnis innerhalb der Gesellschaft. Insofern überträgt er die Überlegungen aus dem Verhältnis Gesellschaft-Gesellschafter mit Ferho auf das Verhältnis Gesellschaft-Geschäftsführer, ohne dies kenntlich zu machen. Dass es sich bei dem Verhältnis Gesellschaft-Geschäftsführer und den daraus resultierenden gesellschaftsrechtlichen Pflichten des Geschäftsführers um einen Vertrag i.S.v. Art. 7 Nr. 1 EuGVO handelt, ergibt sich letztlich nur aus der „freiwillig eingegangenen Verpflichtung“. Es zeigt sich, dass der Gerichtshof auch in diesem Zusammenhang mit einer autonomen Begrifflichkeit („Vertrag oder Ansprüche aus einem Vertrag") arbeitet, bei deren Erfassung er wieder die Unterscheidung von vertraglicher Anstellung und organschaftlicher Bestellung ignoriert. Insofern ist der EuGH konsequent, als dass er die beiden Verhältnisse auch für diese Frage nicht trennt. ${ }^{72}$ Auch hier zeigt sich das autonome Verständnis des Unionsrechts.

\section{Deliktsgerichtsstand}

Mit der Beantwortung der dritten Vorlagefrage stellt der EuGH einen Vorrang der „vertraglichen“ vor der deliktischen Haftung fest: ${ }^{73}$ Eine deliktische Haftung kommt nur in Betracht, wenn der beklagte Geschäftsführer kein Arbeitnehmer im unionalen Sinne ist und kein „Vertrag“ i.S.v. Art. 7 Nr. 1 EuGVO vorliegt. ${ }^{74}$ Während der erste Punkt nur das Ergebnis der zweiten Vorlagefrage für die deliktische Haftung wiederholt, führt der Gerichtshof mit dem zweiten Punkt seine Rechtsprechung aus der Rechtssache Brogsitter ${ }^{75}$ fort. Danach

70 EuGH, Rs. C-34/82 (Peters), BeckEuRS 1983, 105380.

71 EuGH, Rs. C-34/82 (Peters), BeckEuRS 1983, 105380, Rdn. 13; Weller, ZGR 2012, 606, 615 .

72 Krit. von HeIn, IWRZ 2016, 29, 30.

73 LÜtTringhaus, EuZW 2016, 904, 907 („Exklusivitäts- und Vorrangverhältnis“).

74 EuGH, Rs. C-47/14 (Ferho), NZG 2015, 1199, 1203, Rdn. 67, 70.

75 EuGH, Rs. C-548/12 (Brogsitter), NJW 2014, $1648 \mathrm{f} \mathrm{m.} \mathrm{Anm.} \mathrm{Wendelstein,} \mathrm{ZEuP}$ 2015, $622 \mathrm{ff}$. 
bedürfe es für die Anwendbarkeit des Deliktsgerichtsstands gemäß Art. 7 Nr. 2 EuGVO einer Schadensersatzhaftung, die nicht an einen Vertrag „anknüpft “ ${ }^{76}$ Bei Bestehen eines Vertrags müsse der Vorwurf in dem Verstoß gegen vertragliche Verpflichtungen liegen; deren Inhalt sei durch Ermittlung des Vertragsgegenstandes festzustellen. ${ }^{77}$ Eine vertragliche Pflichtverletzung sei dann anzunehmen, wenn eine Auslegung notwendig ist, um die Rechtmäßigkeit des Verhaltens zu klären. ${ }^{78}$

Diese Rechtsprechung ist aufgrund ihrer Konturenlosigkeit nicht ohne Widerspruch geblieben. ${ }^{79}$ Für eine rechtssichere Anwendung der Normen bedarf es weiterer Präzisierung. ${ }^{80}$ Es sind deshalb weitere Vorlagen an den EuGH zu diesen Fragen zu erwarten.

\section{Zusammenfassung in Thesen}

1. Gesellschaften können ihren Geschäftsführer nur vor den Gerichten in dessen Wohnsitzmitgliedstaat verklagen, soweit er unter den unionalen Arbeitnehmerbegriff zu fassen ist. Dies folgt aus der Zuständigkeitskonzentration Art. 22 EuGVO. Mit Ferho überträgt der EuGH seine arbeitsrechtsspezifische Rechtsprechung zum Arbeitnehmerbegriff aus Danosa und Balkaya auf das allgemeine Europäische Zivilprozessrecht (EuGVO). Er konturiert damit zunehmend einen unionalen Arbeitnehmerbegriff, der von dem deutschen Arbeitnehmerbegriff abweicht.

2. Für Geschäftsleiter, die nicht unter den Arbeitnehmerbegriff fallen, gilt regelmäßig der Vertragsgerichtsstand des Erfüllungsorts. Dieser ist anhand des Anstellungsvertrags, der Satzung oder anderer Regelungen zu den Pflichten der Geschäftsleitung oder dem tatsächlichen Tätigkeitsort zu ermitteln.

3. Weiterhin zementiert der EuGH seine Rechtsprechung aus Brogsitter zum Vorrang des Vertrags- vor dem Deliktsgerichtsstand im Fall der Organhaftung im Rahmen der EuGVO, ohne sie weiter zu präzisieren. Weitere Vorlagen zu der Abgrenzung von Art. 7 Nr. 1 und 2 EuGVO sind zu erwarten.

4. Zentrale Voraussetzungen des unionalen Arbeitnehmerbegriffs sind das Weisungsrecht gegenüber dem Leitungsorgan und das Recht zur jederzeitigen Abberufung. Zur Ermittlung dieser Voraussetzungen sind alle Umstände des Einzelfalls zu berücksichtigen. Dazu stellt der Gerichtshof eine Gesamt-

76 EuGH, Rs. C-548/12 (Brogsitter), NJW 2014, 1648, 1649, Rdn. 20.

77 EuGH, Rs. C-548/12 (Brogsitter), NJW 2014, 1648, 1649, Rdn. 24.

78 EuGH, Rs. C-548/12 (Brogsitter), NJW 2014, 1648, 1649, Rdn. 25.

79 Siehe auch von Hein, IWRZ 2016, 29, 31.

80 Lüttringhaus, EuZW 2015, 904, 907; Mankowski, RIW 2015, 821, 823; M. Weller, LMK 2014, 359127; WendelsTein, ZEuP 2015, 622, $632 \mathrm{ff.}$ 
betrachtung aller Rechtsbeziehungen zwischen Gesellschaft(erversammlung) und Geschäftsführer an. Anders als das Trennungsprinzip des deutschen Rechts differenziert er nicht zwischen Anstellungs- und Bestellungsverhältnis.

5. Es wäre aber nicht überraschend, sollte der EuGH den unionalen Arbeitnehmerbegriff auf Art. 8 Rom I-VO erstrecken. Bejaht man die Arbeitnehmereigenschaft im unionsrechtlichen Sinn, erscheint bei konsequenter Fortführung der Rechtsprechung des EuGH eine Doppelqualifikation des Bestellungsverhältnisses als gesellschafts- und arbeitsrechtlich am ehesten geeignet, um alle Interessen sachgerecht zu koordinieren.

6. Im Fall der gesetzlichen Standardkonzeption fällt der Fremdgeschäftsführer der $\mathrm{GmbH}$ unter den unionalen Arbeitnehmerbegriff. Dies gilt nicht für den Gesellschafter-Geschäftsführer, solange er beherrschenden Einfluss auf die Gesellschafterversammlung ausübt, oder auch für die Vorstandsmitglieder einer AG. 